MATEC Web of Conferences 22,02009 (2015)

DOI: $10.1051 /$ matec conf/ 20152202009

(C) Owned by the authors, published by EDP Sciences, 2015

\title{
Analysis of Magnetic Field Intensity and Induced Current under Live Working Based on Charge Simulation Method
}

\author{
Yuanxiang Luo \\ School of Electrical Engineering, Northeast Dianli University, Jilin, Jilin, China \\ Ruiguo Chen \\ State Grid Liaoning Electric Power Supply Company, Shenyang, Liaoning, China \\ Yidong Zhu \\ State Grid Liaoning Academe of Electric Power Science, Shenyang, Liaoning, China \\ Jingui Cheng \\ Xi'an Qin Ping Power Technology Development Limited Company, Xi'an, Shanxi, China \\ Meixia Wei \\ Liaoning Annuoxin Power Technology Limited Company, Xi'an, Shanxi, China
}

\begin{abstract}
To the problem that safety distance is insufficient for $500 \mathrm{kV}$ substation live working, a magnetic field analysis method for overhead line bus is given based on the charge simulation method. In the method, charge is calculated firstly, and the space field intensity distribution calculation is completed by overlying charge. The space field intensity distribution rule is carried out based on the appropriate analysis, and space field intensity distribution rule of substation is obtained. Then according to the calculation formula of inducing current, the human body induction current under a substation busbar is simulated based on MATLAB. The simulation results have a certain guidance function for actual live working.
\end{abstract}

Keywords: live working; charge simulation method; field intensity distribution; induced current

\section{INTRODUCTION}

As an important skill of power transmission and transformation industry, charge detection plays an important role in improving power supply reliability of power transmission, transformation system and especially important substation [1]. But high density and electromagnetic fields in charged body under live working do harm to live working personnel physically and psychologically. In the dense decorated substation, in order to save investment and save floor space, it may appear that the electromagnetic field density is higher, and may bring greater harm. So before living working, it is necessary checking on safety distance and carrying out electromagnetic field analysis.

About substation and overhead line analysis, a lot of experts and scholars have done detailed research on it. And a series of research results can be obtained. In [2], power frequency electromagnetic field distribution of the certain northeast substation equipment is measured through the measured method. In [3], the electric field intensity of a single transmission line is analyzed using charge simulation method. It is used for the analysis of human body inducing current, and good results have been achieved. In [4-5], the field strength distribution of horizontal and vertical conductor is based on dipole field theory.
In this paper, the conclusion is different from charge simulation method. It says that field size is proportional to the current size, rather than voltage. It provides a new perspective for the field intensity analysis theory. In [6-7], the human body induction current distribution under the overhead based on numerical analysis method makes a detailed discussion on distribution rule of human body induction current In [8], the induced current of the body is calculated using the simplified calculation formula proposed by the international conference on power grid (CIGRE) 36.01 group.

In this paper, taking the measured parameters of a $500 \mathrm{kV}$ substation as the prototype, on the basis of the prototype, appropriate simplification is made. The corresponding simplified harmonic analysis model can be obtained.

The corresponding space field intensity distribution can be obtained based on the simplified analysis model. Using the steps above to obtain field intensity and human body induced current is calculated based on the simplified induced current calculation formula. And human body induced current affected by space position is analyzed. 


\section{ELECTRIC FIELD INTENSITY ANALYSIS} THEORY

\subsection{Calculation principle of line charge}

Usually the slender conductor is equivalent as the line model. The voltage and current are generally known as the quantity of wire conductors in engineering, but analysis of electric field intensity needs charge density. So we need to study the relationship between charge density and voltage or current, which usually means the relationship between voltage and line charge density as follows:

\section{$\mathbf{U}=\mathbf{P} \tau$}

Where, $\mathbf{U}$ is $n \times 1$ order voltage vector, $\mathbf{P}$ is $n \times n$ potential coefficient matrix, and it can be obtained based on the principle of the image. ${ }^{\tau}$ is $n \times 1$ order line charge density vectors.

All that said, usually voltage vector is known, so Equation (1) needs to be appropriately transformed to:

$$
\boldsymbol{\tau}=\mathbf{H U}
$$

Where, $\mathrm{H}=\mathrm{P}^{-1}$.

Then we need to discuss clear expression given by potential coefficient matrix. According to the principle of mirror image, it is not difficult to obtain expression of the main diagonal and other position in $\mathbf{P}$ :

$$
p_{i j}=\left\{\begin{array}{l}
\ln \frac{2 h_{i}}{R_{i}}(i=j) \\
\ln \frac{r_{i, j}^{\prime}}{r_{i, j}}(i \neq j)
\end{array}\right.
$$

Where, $h_{i}$ is the height of the wire $i$ to the ground, and wire is a single phase conductor; $R_{i}$ is the radius of the wire $i$; When the wire is a split conductor, $R_{i}$ is the equivalent radius; $r_{i, j}$ is the distance between the wire $i$ th and wire $j$ th; $r_{i, j}$ is the distance that wire $j$ is from the wire $i$.

According to the Equation (2), the simulation of electrical charge and numerical mirror charge can be obtained.

Suppose there are $n$ simulation charge, $\boldsymbol{\tau}$ is $n * 1$ column, $H$ is $n * n$ phalanx, and $U$ is $n * 1$ column. To solve the analog electrical charge, the image electrical charge can be converted into the problem $n$ that linear equations are to be solved.

But it still exists in a kind of constraint and special field with known points (such as ground and ground wire) which need to be added into Equation (1), and they are called matching points. The augmented matrix added into the matching point is called (4) :

$$
\mathbf{U}^{\prime}=\mathbf{P}^{\prime} \boldsymbol{\tau}^{\prime}
$$

Where, $\mathbf{U}^{\prime}=\left[\begin{array}{ll}\mathbf{U} & \mathbf{0}\end{array}\right] ; \boldsymbol{\tau}^{\prime}$ is the line charge density of matching point;

$$
\mathbf{P}^{\prime}=\left[\begin{array}{cc}
\mathbf{P} & \mathbf{P}_{12} \\
\mathbf{P}_{21} & \mathbf{P}_{22}
\end{array}\right],
$$

$\mathrm{P}_{12}$ is the line charge of simulation point and potential coefficient matrix of the matching point; $\mathrm{P}_{21}$ is line charge of matching point and potential coefficient matrix of simulation point; the line of the matching point $\mathrm{P}_{22}$ is the line charge of matching point and the potential coefficient matrix of matching point, $\mathbf{U}^{\prime}=\left[\boldsymbol{\tau} \tau^{\prime}\right]$; , and $\boldsymbol{\tau}^{\prime}$ is the linear charge density of the matching point.

From Equation (3) in inverse processing, the density of simulation charge and matching charge can be obtained as follows:

$$
\boldsymbol{\tau}^{\prime}=\left(\mathbf{P}^{\prime}\right)^{-1} \mathbf{U}^{\prime}
$$

\subsection{The calculation of electrostatic field strength} under the action of line charge

According to the theory of electrostatic field, the expression of the relationship between electric field intensity and the line charge density of infinite long conductor wire in the y axis parallel to the direction can be shown in the following:

$$
\left\{\begin{array}{l}
E_{x}=\frac{1}{4 \pi \varepsilon} \sum_{i=1}^{n} \tau_{i}\left(\frac{x-x_{i}}{R^{2}}+\frac{x_{i}-x}{R^{\prime 2}}\right) \\
E_{z}=\frac{1}{4 \pi \varepsilon} \sum_{i=1}^{n} \tau_{i}\left(\frac{z-z_{i}}{R^{2}}-\frac{z+z_{i}}{R^{\prime 2}}\right)
\end{array}\right.
$$

Where, $n$ is the number of phase of power transmission lines; $x_{i}$ and $z_{i}$ are the coordinates of the equivalent electric charge respectively; $x$ and $z$ are the coordinates of calculating point respectively; $R$ is the distance between calculating point and image electrical charge.

Combing with Equations (5) and (6), the expression of electric field intensity about voltage can be concluded that:

$$
\left\{\begin{array}{l}
E_{x}=\frac{1}{4 \pi \varepsilon} \sum_{i=1}^{n}\left[\left(\frac{x-x_{i}}{R^{2}}-\frac{x-x_{i}}{R^{\prime 2}}\right) \sum_{l=1}^{m} h_{l} u_{l}\right] \\
E_{z}=\frac{1}{4 \pi \varepsilon} \sum_{i=1}^{n}\left[\left(\frac{z-x_{i}}{R^{2}}-\frac{z-x_{i}}{R^{\prime 2}}\right) \sum_{l=1}^{m} h_{l} u_{l}\right]
\end{array}\right.
$$

Where, $m$ is the dimension of $H$ matrix. $U_{l}$ is the voltage vector elements. 


\section{CALCULATION OF INDUCED CURRENT}

When people are in a $500 \mathrm{kV}$ substation charge operation, a certain induction voltage is produced additionally. The induction loop is likely to be formed between the line and earth, and it forms a certain induced current. It is more significant for current than the induced voltage to impact on the human body. Thus it is necessary to make reasonable assessment on the induced current when live working, so as to ensure the safety of live working personnel.

Based on Equation [4], simplify the induced current calculation method when upright body in power frequency electromagnetic field can be obtained as follows:

$$
I(t)=\pi \omega \varepsilon_{0} E\left(2 h_{1}^{2}+h_{2}^{2}\right)
$$

Where, $I$ is induced current, and the unit is $\mu \mathrm{A} ; f$ is frequency, and the unit is $\mathrm{Hz} ; \omega=2 \pi \mathrm{f}$, which is angular frequency, and the unit is $(\mathrm{rad} / \mathrm{s}) ; \varepsilon_{0}$ is the dielectric constant, and the value is $10^{-9} / 36 / \pi ; h_{l}$ is head height, and $h_{2}$ is trunk height.

\section{EXAMPLE ANALYSIS}

Line height is $9 \mathrm{~m}$ from the ground, and the distance of different phase conductor is $4 \mathrm{~m}$. The bus voltage level is $500 \mathrm{kV}$.

\subsection{Analysis of field intensity distribution of line}

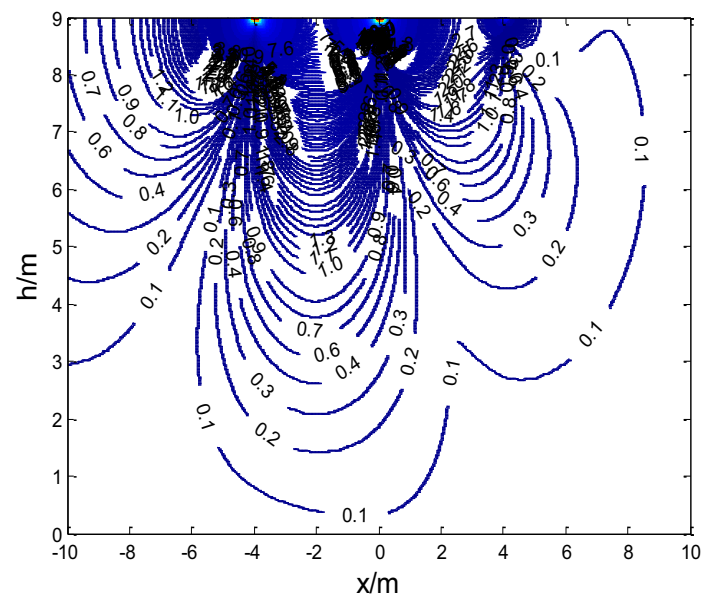

Figure 1 . The $a$ phase distribution of field intensity when the phase is $0^{\circ}$

It can be shown in Figures 1-4, the space field intensity constantly attenuates, with three buses as the core The field intensity from $2 \mathrm{~m}$ height to ground is vertical distribution. And it is evident distortion of field strength around $2 \mathrm{~m}$ radius of conductor. The $4 \mathrm{~m}$ to $5.7 \mathrm{~m}$ height in $Y$ direction attenuates, that is, field strength in the range of live working does not obviously attenuate. In China, occupational exposure of the electric field intensity that is limited by the power frequency electromagnetic field exposure rule is $6 \mathrm{kv} / \mathrm{m}$. Obviously, under the condition of each phase, the electric field intensity does not exceed this limit. The field intensity change trend is shown in Figures 1-4, and the field strength that is placed in area from $4 \mathrm{~m}$ to $5.7 \mathrm{~m}$ shown in Figure 1 and Figure 3 is weak. The dense regions of field lines are mainly concentrated in the area of more than $6 \mathrm{~m}$, and $a$ phase is equal to $0^{\circ}$. The maximum electric field intensity density area lies in between $a$ and $b$ phase, and $a$ phase is equal to $180^{\circ}$. The maximum electric field intensity density area is between $b$ and $c$ phase. When a phase is equal to $90^{\circ}$ and $270^{\circ}$, regional field strength in $4 \mathrm{~m}$ and $5.7 \mathrm{~m}$ is stronger. But the electric field line is relatively sparse. Figure 2 and Figure 4 similarly lie in the center of the field which is located in the $b$ phase.

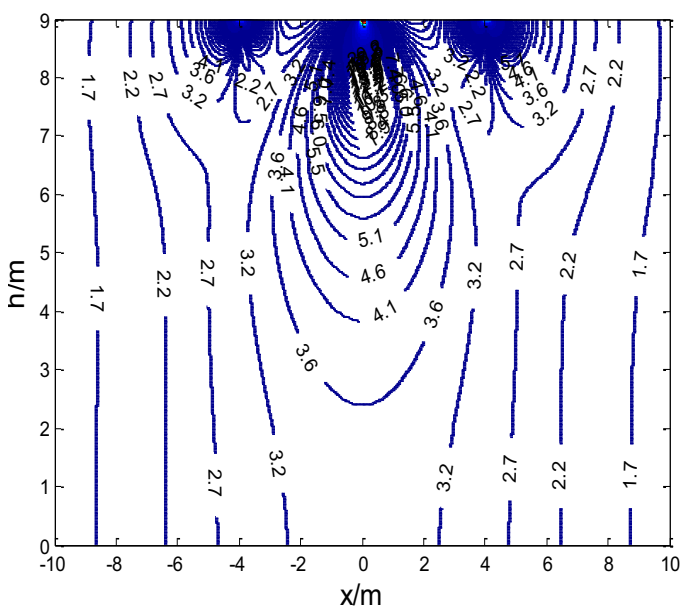

Figure 2. The $a$ phase distribution of field intensity when the phase is $90^{\circ}$ 


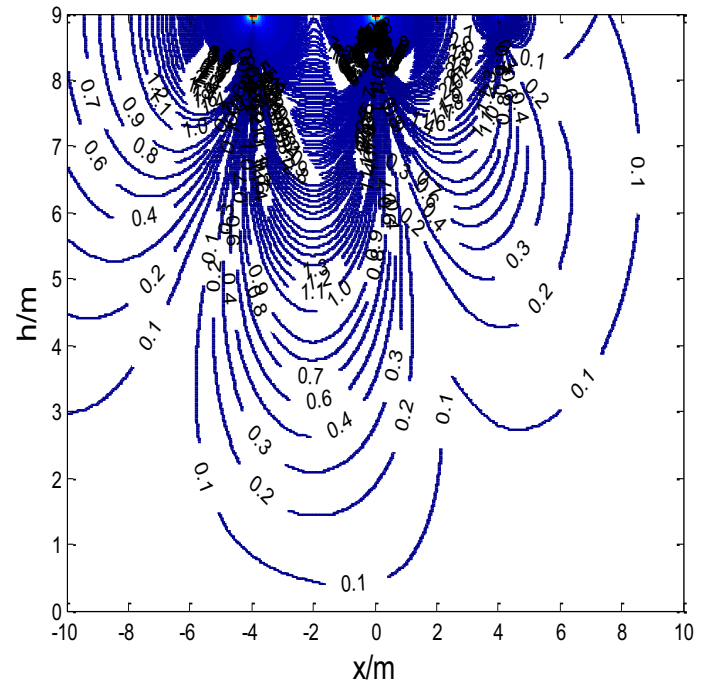

Figure 3 . The $a$ phase distribution of field intensity when the phase is $180^{\circ}$

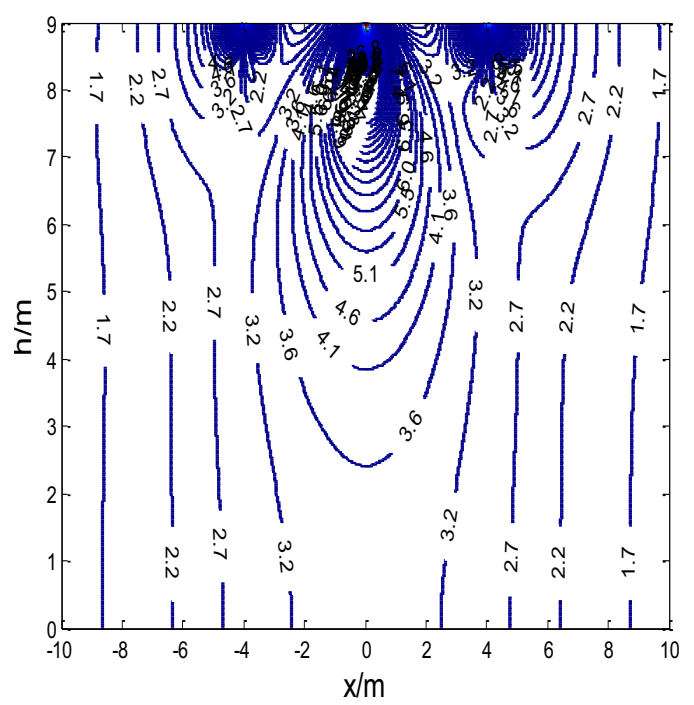

Figure 4. The $a$ phase distribution of field intensity when the phase is $270^{\circ}$

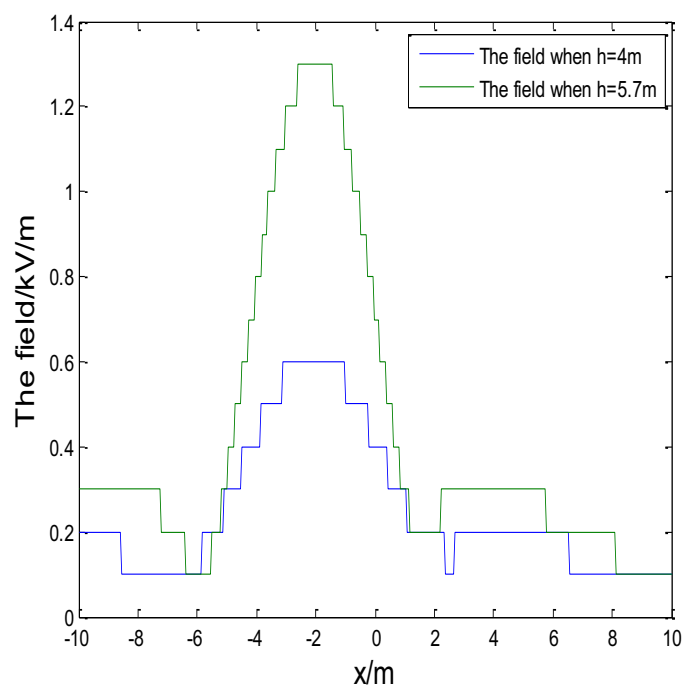

Figure 5. A field intensity distribution of different heights when the $a$ phase is $0^{\circ}$

And obvious field intensity changes exist in relatively sparse segmented regions among the three phases. As shown in field intensity change trending from Figures 1-4, maximum density area of the electric field changes with phase. It proves that the field intensity produced by the alternating current (ac) is time-varying.

When live working, workers need to stand on the $4 \mathrm{~m}$ high insulation. As the average height of the human body is $1.7 \mathrm{~m}$, it is estimated that person's head is about $5.7 \mathrm{~m}$ height to the ground. So in this article, two representative heights that are $4 \mathrm{~m}$ and $5.7 \mathrm{~m}$ are taken to estimate the space field intensity.

Because the field strength is continuous, so according to the field intensity of the head and foot height, whether the field strength of the head and foot in the middle area is overweight can be estimated. 


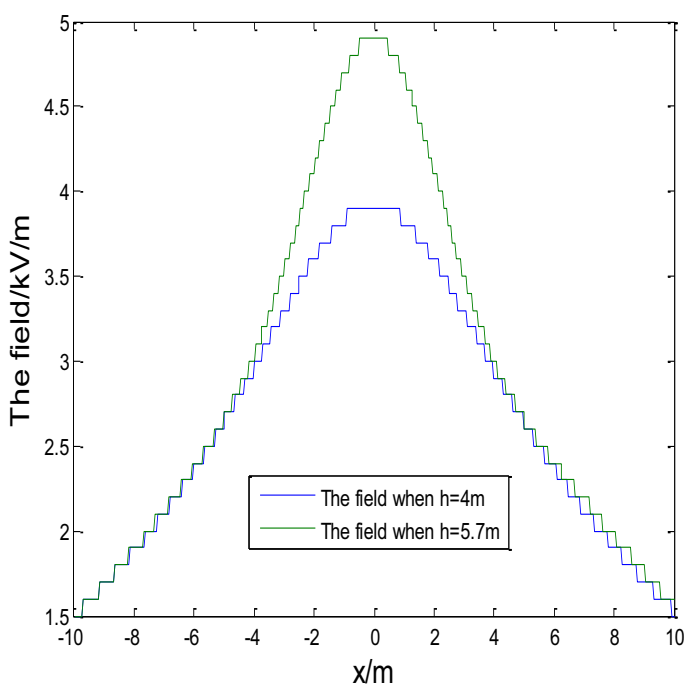

Figure 6. A field intensity distribution of different heights when the $a$ phase is $90^{\circ}$

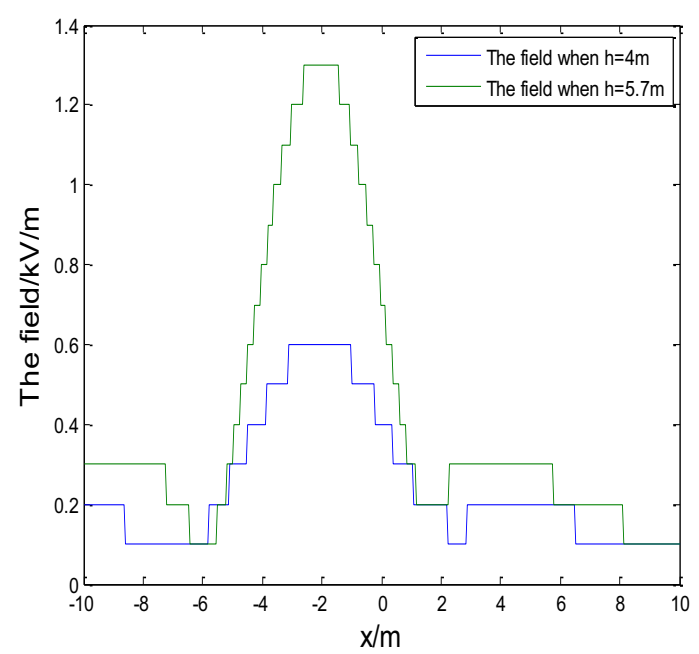

Figure 7. A field intensity distribution of $4 \mathrm{~m}$ and $5.7 \mathrm{~m}$ height when $a$ phase is $180^{\circ}$

As we can conclude from Figures 5-8, the field strength on the other side circuit below line also changes with the phase angle. The maximum field strength occurs near phase angles of $90^{\circ}$ and $270^{\circ}$.

But field intensity distribution of $90^{\circ}+\mathrm{k}^{*} 180$ and $0^{\circ}+\mathrm{k}^{*} 180$ is slightly different. The field intensity distribution on the level surface of $90^{\circ}+\mathrm{k} * 180$ is a single peak. The field intensity distribution of $0^{\circ}+\mathrm{k}$ * 180 is different. On the other hand, in addition to the peak of the center, there are two small peaks on both sides. The energy distribution of $0^{\circ}+\mathrm{k} * 180$ is con- sistent more than $90^{\circ}+\mathrm{k}^{*} 180$

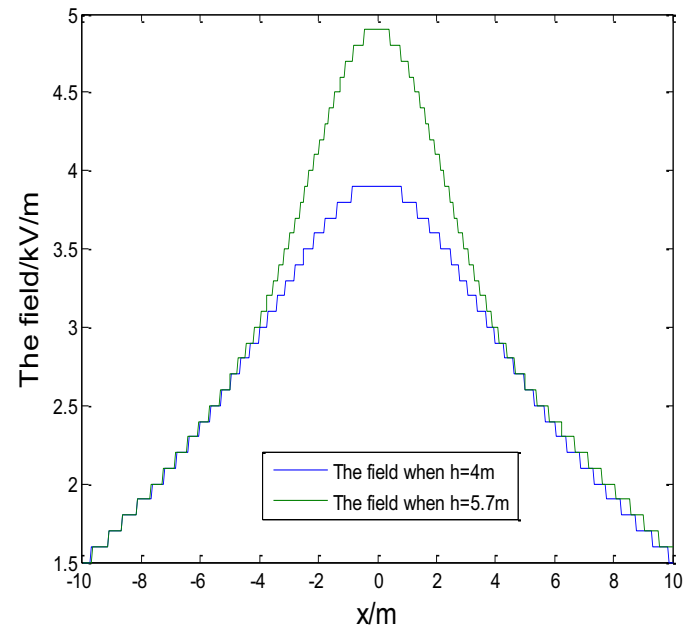

Figure 8. A field intensity distribution of $4 \mathrm{~m}$ and $5.7 \mathrm{~m}$ height when $a$ phase is $270^{\circ}$

\subsection{Analysis of induced current of human body}

Taking $1.5 \mathrm{~m}$ as human body height, and the head height is $0.2 \mathrm{~m}$. The electrical field strength changes with the position.

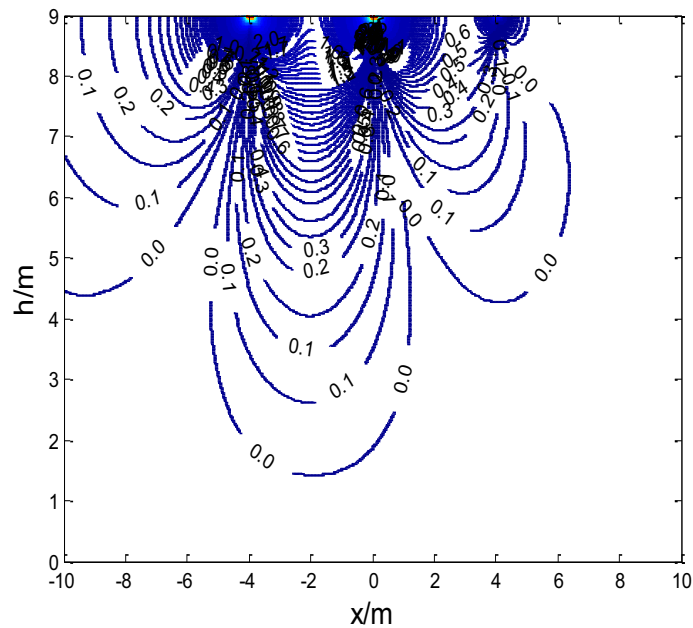

Figure 9. The body induction current distribution $(\mathrm{mA})$ of $500 \mathrm{kV}$ bus when the $a$ phase is $0^{\circ}$

As we can see from Figures 9-12, inducted current law is similar to the field strength rule, and the human body current is $\mathrm{mA}$ level. According to guide rules that limit AC power field, magnetic field and electromagnetic field are published by the international commission on non-ionizing radiation protection. And the fundamental limit value of the current density of professional personnel is $10 \mathrm{~mA} / \mathrm{m}^{2}$. Human skin area 
is about $3 \mathrm{~m}^{2}$. So according to the data shown in Figure 3 , the induced current of human body in the working environment is safe.

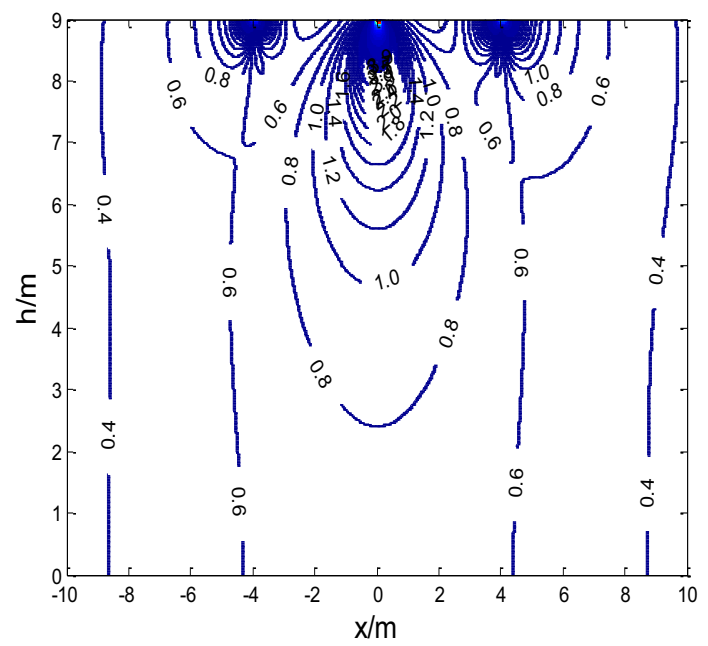

Figure 10. The body induction current distribution $(\mathrm{mA})$ of $500 \mathrm{kV}$ bus when the $a$ phase is $90^{\circ}$

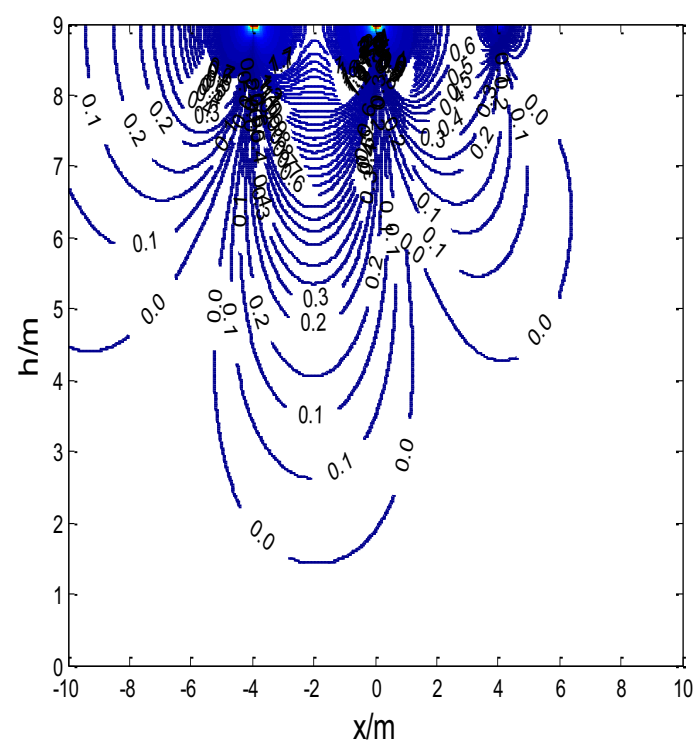

Figure 11. The body induction current distribution $(\mathrm{mA})$ of $500 \mathrm{kV}$ bus when the $a$ phase is $180^{\circ}$

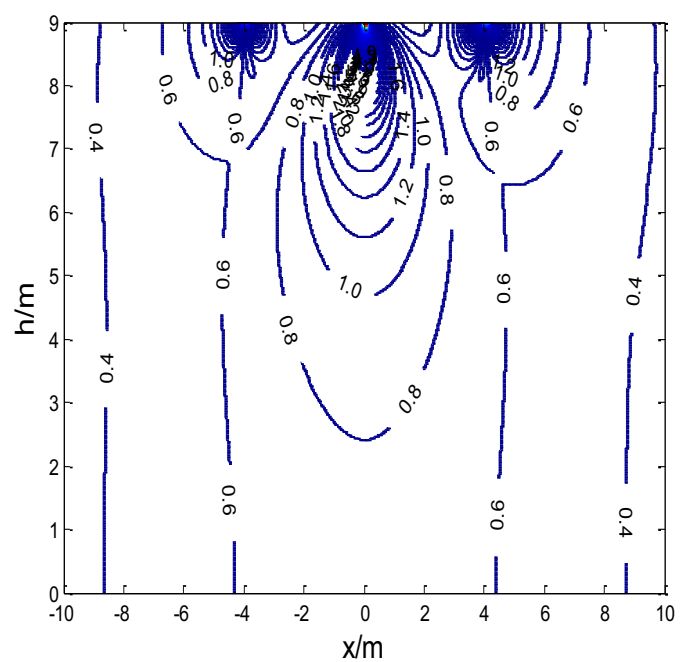

Figure 12. The body induction current distribution $(\mathrm{mA})$ of $500 \mathrm{kv}$ bus when the $a$ phase is $270^{\circ}$

\section{CONCLUSIONS}

In this paper, the field and induced current analysis of problems are carried out based on charge simulation method. This method has the advantage that the field strength analysis and induction current laws can be seen intuitively. And on the condition of computation it can be accepted that high accuracy can be obtained. The problems which need to be further discussed are:

(1) As to local eddy current problems of the human body, more sophisticated numerical simulation method is needed to be verified. Especially, as to local induced current intensive area, it should be more focused on. In addition, the various parts of the body to stand the strength of the induced current are also different. So each part of the induction current needs to differentiate with consideration and analysis.

(2) Although two-dimensional spatial distribution is studied, the need to study the distribution of the three-dimensional space, further expansion of the field intensity distribution and the change of the induced current is urgent. Especially, the location of the lowest body endurance value [9] and induced current peak of the third dimension are focused on.

\section{REFERENCES}

[1] Hu Yi, Liu Kai. \& Peng Yong. et al. 2014. Research status and development trend of live working key technology. High Voltage Engineering, 40(7):1921-1931.

[2] Rao Zhangquan. \& Guo Qigui et al. 2004. Measurement of power frequency electric \&magnetic field in $500 \mathrm{kV}$ substation. High Voltage Engineering, 9(30):41-43. 
[3] Yang Wenhan. \& Lu Yinghua. 2008. Application of emulation charge method in calculation of electromagnetic environment near to HV transmission lines. Power System Technology, 32(2): 47-50.

[4] Zheng Jie, Lin Tao. \& Bi Yue et al. 2010. An approach to calculate electric field strength produced by AC currents flowing through vertical conductor, Power System Technology, 34(6): 19-22.

[5] Zhao Peng, Cui Dingxin. \& Qu Xuedi. 2010. An approach to calculate electric field strength resulting from AC currents flowing through horizontal infinite line, Power System Technology, 34(6):23-26.

[6] Zishan Tian. 2013. A thesis submitted to Chongqing University in partial fulfillment of the requirement for the master's degree of engineering. Electrical Switching, 5 .

[7] Wang Jianhua, Wen Wu. \& Ruan Jiangjun. 2007. Calculation and analysis on power frequency magnetic field induced current density within human body under UHVAC transmission line. Power System Technology, 31(13): 7-10+33.

[8] Cui Dingxin Qu Xuedi. 2012. Evaluated Calculation of the Induced Current in Human Body Produced by Power Frequency Electric and Magnetic Field under $A C$ Transmission Line. Southwest Jiaotong University, 6.

[9] Yu Mengting, Wang Jingang, \& Li Jian. 2013. The experimental study on the human body influence on measurement of high voltage power frequency Electric Field.

Electrical Measurement \& Instrumentation, 5(60): 24-27(48). 